\title{
An Architecture Model for Optimal Allocation of Water to Domestic Users using Data Mining Techniques - A Case Study of Bengaluru City
}

\author{
Sara Kutty T. K. \\ Research Scholar \\ Rayalaseema University \\ Kurnool, India
}

\author{
M. Hanumanthappa \\ Department of Computer Science and Applications \\ Bangalore University \\ Bengaluru, India
}

\begin{abstract}
Various economic sectors like municipal, agricultural, industrial, commercial, hydropower, recreation and environment depends on surface water from rivers, lakes, reservoirs, groundwater and flood-water. Due to the increase in population growth rates, increased population density, improved life style, pollution, industrial expansion, spatial distribution of urban and industrial requirements water resources has become infrequent. The growing demand for water cannot be met with the conventional methods; therefore it is very important to focus on water conservation and to allocate water more efficiently and economically. Water demand and supply corresponding to environmental constraints, social preferences, pricing system, and development priorities are the major deciding factors for water allocation. Huge volumes of water data are available and to handle this vast volume of water data, data mining techniques are used. The inputs from water data combined with computational data mining techniques helps in building models capable of optimal allocation of water. The focus of water allocation model is to evaluate the amount of water used by domestic users and to optimally allocate water to them. In this paper residential water demands was considered to design the model for equivalent residential units. An architecture model using differential evolution algorithm has been proposed for optimal allocation of water to different domestic users in particular to Bengaluru city.
\end{abstract}

\section{Keywords}

Data Mining, Optimal Allocation, Architecture Model, Differential Evolution Algorithm.

\section{INTRODUCTION}

Olden days urban water supply systems, was designed and planned on least-cost approaches but nowadays the focus is more on factors related to environmental sustainability [1]. The consumption of water increases as the standard of living increases. The ever increasing demand for water cannot be met because of the ever increasing population growth, limited water resources, improper management of water resources, industrialization, urbanization, improper supply of available water resources. Traditionally much importance was not given for the amount of water consumed after water distribution. The ratio between consumption and accumulation based on the current data is important to estimate and forecast gross domestic requirement [2]. Per capita water availability is declining on a daily basis because of the limited availability of fresh water, population explosion and high water consuming economic activities. The Bureau of Indian Standards has laid down the rules for water consumption. But always it is not possible to meet this because of the ever increasing needs of users and increase in population. Some of the issues related to the scarcity of water are improper water supply, water financing, equity and social justice, pricing, governance and management. Each country has a unique system for allocating the available water based on the demand. The water allocation model of each country or city varies based on the water resources infrastructure and the institutional and legal frameworks within the country.

The process of extracting implicit, potentially useful or previously unknown information from the data is called knowledge discovery. Figure 1 depicts the knowledge discovery process. Data collected from different sources is integrated into a single data store called target data. Preprocessing is done on this data and it is converted to the standard format. The data is processed using data mining algorithms to new or useful knowledge or information. Extracting and interpreting the hidden patterns from a large amount of data is called data mining. Data mining handles large volumes of data with traditional data analysis method and sophisticated algorithms.

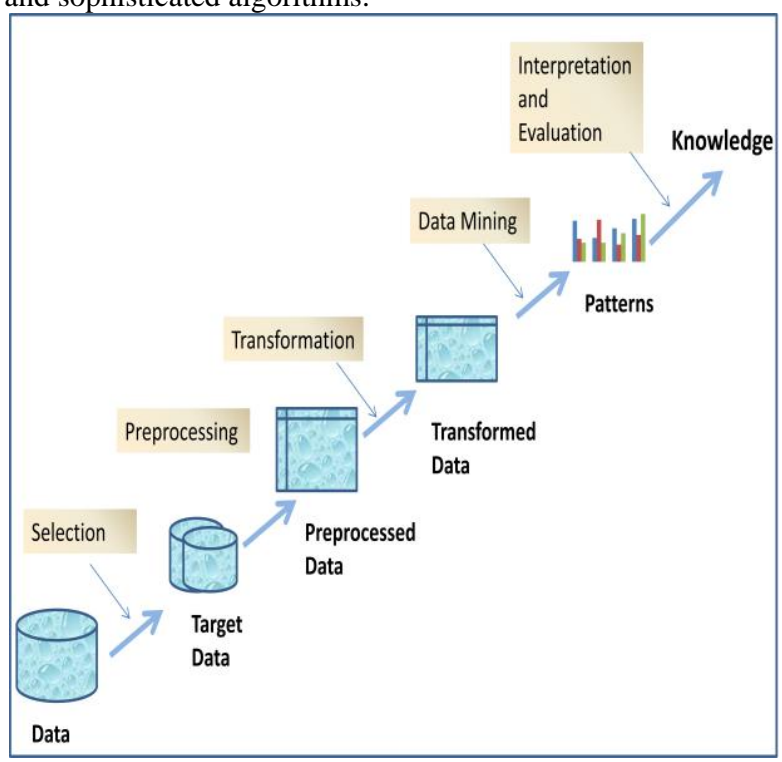

Figure 1: Knowledge Discovery Process

Data driven models using data mining techniques is widely used in water allocation problems to optimally allocate water, to calculate water demand, to forecast future water requirements, to increase water productivity and for proper management of water resources. Some of the data mining techniques used in water resource management are association rule learning, classification analysis, clustering analysis, anomaly or outlier detection, and regression analysis. 
Classification analysis helps in classifying data into different classes. In order to build relationship among different datasets, forecast and to examine user behaviors association rules are used. For fault detection, fraud detection, intrusion detection anomaly or outlier detection is used. Clustering is used to find groups or clusters in the data. For prediction and forecasting regression analysis is used.

In this model first the data is collected and pre-processed. Preprocessing is done because the available data was inconsistent due to redundant data, variations in data format, missing data and unrealistic data. It helped in integrating, cleaning, electing the most significant attributes and preparing the data in useable format before performing any mining. On this pre-processed data the architecture model is built [4].

This paper is organized as follows, section 2 contains a literature survey of various water allocation problems and describes the study area, section 3 describes the water allocation model and section 4 concludes the paper with some suggestions for future work.

\section{LITERATURE SURVEY}

Shivshanker Singh Patel and Parthasarathy Ramachandran has discussed how integrated water management using a novel nonlinear optimization model and policy analysis can be used to ensure the basic water needs with the available surface water resources [5]. Stephen X. Zhang and Vladan Babovic assessed innovative water technologies to provide decision support system under uncertainty [6]. Environmental, social and political factors influence water allocation and management. Water allocation based on economic criteria or on environmental needs was the two main factors considered during the past. Socio-economic, environmental, technical aspects, ever changing demand and supply was not considered in developing water allocation models. Economic efficiency, technical feasibility and socially fair allocation are the important points to be considered for allocation of water [7]. Researchers have developed many models for optimized water allocation, but these models aimed at maximizing the economic returns based on water usage. The demand coefficient was not there prime aim. For a sustainable water allocation system water has to be allocated based on the demands. Data mining techniques like dynamic programming, genetic algorithms, and game theoretical approach have been applied to water allocation problems [8]. For Jiangxi province of China, Ling and Gui [9] used simplex method and genetic algorithm to allocate water resources optimally. A simulated water allocation model was developed by Green and Hamilton for irrigation efficiencies, consumption, and flow and shortage rights. Babel et al. [7] used SICCON technique to develop a multi-objective water allocation model to optimally allocate water for a hypothetical reservoir. Considering environmental, social and economic criteria's Roozbahani et al. [10] designed a multi-objective optimization program model. Ahmad et al. [8] used ROM and BLMOLP for the optimal water allocation under the hierarchical model to the Swat River basin of Pakistan. J JYOU et al. developed a ROWAS model which had three layers the first layer was used to allocate water in time scale; the second layer to allocate water in spatial scale and the third layer to distribute water in various sectors [2].

The urban water system infrastructure consists of provision or abstraction, treatment or purification and distribution or end use. In provision raw water from a source like lake, river, groundwater etc. is extracted and then pumped. Then the raw water is purified to drinking water quality standards through filtration, flocculation, and disinfection so that it meets the quality standards prescribed by the Bureau of Indian Standards. This purified water is transported to the end users (domestic customers) through pumping and pressurization [1]. Domestic users collect this water in sumps and use it on-site. The challenges faced by water management are to meet the water demands of variety of users and to allocate it optimally with limited loss. Therefore water allocation plays an important role in water management. There is no standard benchmark for the requirement of water for domestic use. A per capita water supply of 90-100 liters per day is the requirement according to the National Commission on Urbanisation (quoted in Ramachandraiah, 2001).

Considering the different factors the average requirement per household is $135 \mathrm{lpcd}$. Bengaluru city depends on water from Cauvery river with a gross of 1,410 million litres a day (MLD). For one crore population of Bengaluru at present the allocation is only 90 litres per capita per day whereas the rules of government of India says 150 lpcd for metropolitan cities. Different users obtain water from a single master water meter from the utility supplier (Ex: BWSSB in Bengaluru), or extracted from bore-wells within the site. Currently Bengaluru city's water demand is 1500 MLD. Out of this $60 \%$ is taken care by BWSSB and the rest from groundwater via bore-well, tanker, etc. Groundwater levels of Bengaluru are exhausting, so different water conservation methods like usage of harvested rain water; recycling of waste water, lake rejuvenation, controlled usage of water through pay as you use has to be used in order to save this precious commodity.

In cities like Bengaluru the supply of water is a big challenge because of the diminishing water resources, fast increase in population and greater consumer needs. A study on the quantity of water consumption in domestic households like different sources of water supply and duration of corporation water supply in Bengaluru city was carried out. Water requirement of each household is based on different criteria's like food habits, climate, work, culture, working conditions, physiology etc. $92 \%$ of Indian cities depend on municipal water supply to fulfill their water demands. And some households do not depend on water from only one source but from multiple sources. Both in the urban and rural areas the demand for drinking water demand is increasing quickly. Therefore, in the coming years we have to take special precautions to save this precious commodity - water. Therefore water allocation for larger population has to depend on multiple sources like perennial rivers and reservoirs, ground water, rain water harvesting and usage, treated water etc. Improper water allocation strategies have resulted in inequitable distribution of water and excessive use of water. Bengalurians pay the highest price for drinking water in India and in Asia. Also Bengaluru is the lone city with 100 percent metering in Asia. Bengaluru city has bagged several national awards for its efficient handling of various components of urban water management (Sastry, 2006). Even with 100 percent metering, the unaccounted water is one of the highest being 40 percent. Thus there is an urgent need for reducing this large proportion of unaccounted water [11]. In Bengaluru water on tap is not available 24 hours a day. Once in two days the municipal corporation supplies water through piped networks which is stored in tanks, sumps and drums. For further needs people depend on water tankers and bore-wells.

\section{MODELLING FRAMEWORK}

Olden days water allocation was to distribute water to different regions and sectors, without enough consideration of the amount of water consumed after water distribution. Water allocation should focus on the following criteria's; security, 
equity, real opportunity cost, flexibility, predictability, efficacy, political and public acceptability, administrative feasibility and sustainability. Optimal allocation of water can be equity based or user defined priority based. Equity based allocation focuses on fairness of water allocation across economically disparate groups. In equity based allocation water is allocated based on the number of people in each building. It does not consider the different needs of different users and uses. The user defined priority can either be single or multiple priorities, where the user can make the decision based on the usage of water from bore well or the use of harvested rainwater [8].

The architecture model has been designed for optimally allocating water to different domestic users in particular to Bengaluru city. Figure 2 depicts the architecture model for water allocation which consists of the inputs, key modules, and outputs. The reckoning is carried out building wise and then combined appropriately for group of buildings and then city level. Per capita water consumption, population information, the ratio of industrial/domestic consumption is some of the data considered in the model development. This model is capable of estimating the water needs of the existing and projected population and can model the water allocation conditions in a particular area.

The model can be used to access the performance and functionally of the water system within a specific area. In general a model consists of the following components; parameters, variables and constraints. Parameters represent numerical values that are fixed. Variables represent the behavior and the performance of the system being modeled. Constraints represent the relationship of the system's operation on the parameters and variables. The architectural diagram consists of the following modules; water database, water demand module, water availability module, water allocation module, water balance and optimal allocation module. The model was constructed in such a way that it reduces the water use and loss and all domestic users get a reasonable amount of water. The details of the estimation, inputs, assumptions and key equations used in the modules are described in the following subsections.

\subsection{Sources of Water}

The water allocation model was based on the baseline plan for the city of Bengaluru. In the baseline plan, it is expected that domestic users receive water from BWSSB and also uses water from bore wells and rain water harvesting. The water received from BWSSB is the public water source and the water obtained from bore wells and rain water harvesting is considered as independent water sources. The sewage water treatment was not considered because it is not successfully implemented in the city. In future it can be incorporated into this module. Variations in water demands based on seasons, availability, water loss due to leakage, pump failure are not considered because it is not feasible for BWSSB to incorporate this in water supply system. Present supply of water to Bengaluru city is from Cauvery River. In addition to these households depends on water from rainwater harvesting, bore wells, public taps and water tanker Lorries. The present supply of water to Bengaluru city from Cauvery source is 1350 MLD. This has to be distributed to 8.65 lakhs house service connections. The inputs to the model are buildinglevel description of the municipal area, number of occupants in each building, water source options for each building like bore well, usage of harvested rain water.

\subsection{Water Database}

The heart of the water allocation model is the water data base which consists of all the data related to water allocation like year wise consumption of water, category wise consumption, population and water supply requirements, different water availability scenarios and demand forecasts. Intermediate storage between the modules and communication between them is possible through this water database. Water database is used to find the availability of water resources, managerial and infrastructural options, water demand and developmental policies to be formulated within the scope of the analysis.

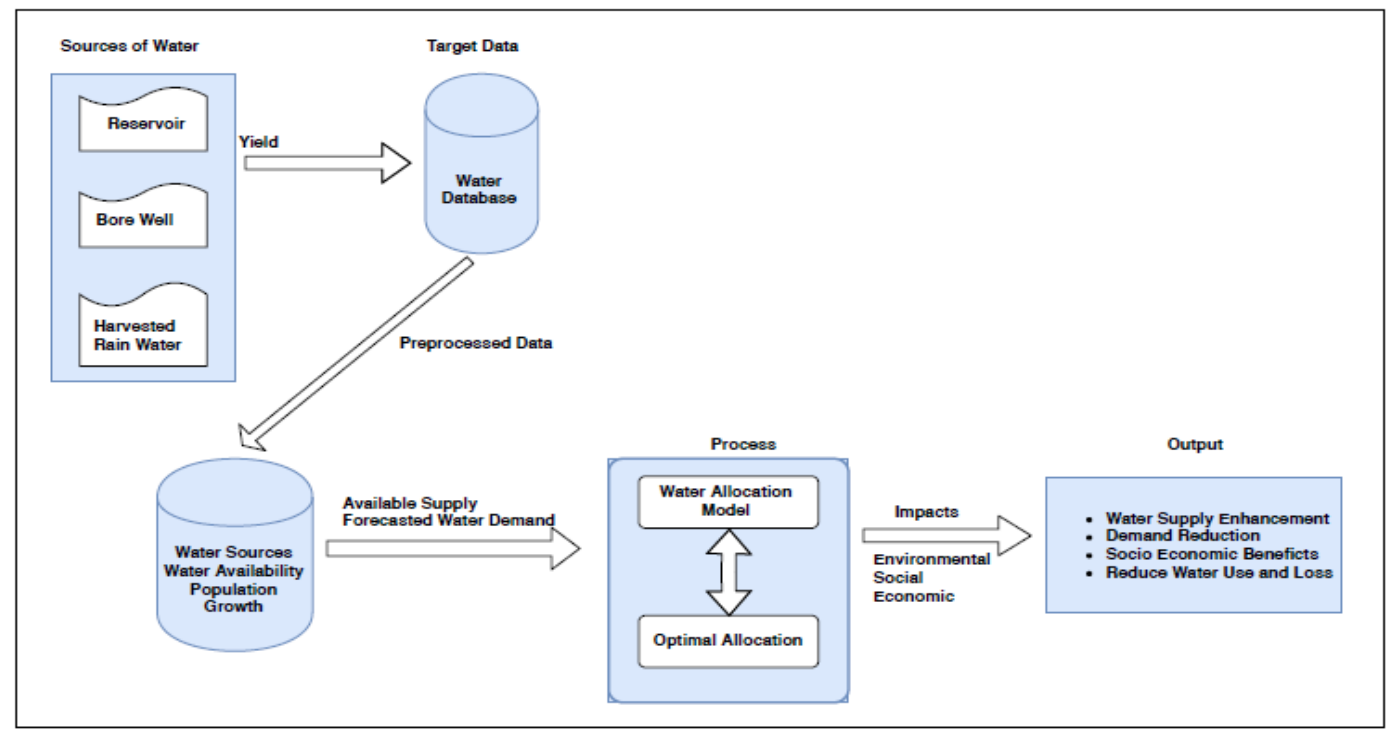

Figure 2: Architecture model for optimal water allocation

The core modules of the data model are basic regional data which contains the general information of the case study area like plot number, plot area in sqft, number of floors, usage type, number of occupants, bore well usage, quantity of bore well water usage in lpcd, rain water harvesting, usage of harvested rain water in lpcd. While considering water demand data we should remember that water usage patterns are usually unique because of the individuality of consumers on the system, their expectations to be able to use water whenever and however they wish, and how well community, 
cultural, or societal desires influence or impact these individuals. Water demand limits may be achieved aversely if most users have a common goal on water use, which does not happen always.

\subsection{Water Allocation Module}

In the allocation model the elements are represented as water nodes and the water links are the connections between them. The different categories of water nodes are supply nodes, demand nodes and transshipment nodes. The supply node represents the water supply. Water to users is provided through the outgoing links and as ingoing links. Outgoing links are expressed as water source function and ingoing links are reservoirs, bore wells (ground water), rain water harvesting (artificial tanks built to collect rainfall water). Demand nodes, models water uses and flow requirements. Demand nodes are settlement which conceptualises the civil urban population and infrastructures of a defined area that is a city, a town or a village, industrial site, irrigation site, environmental, recreation and navigation. Here we focus only on settlement. Transshipment nodes, represents treatment plants and generic network junctions used for maintaining network connectivity and acts as an intersection point between links. Water link objects are classified into different categories based on the connectivity rules of the network and the particular modeling requirements. Some of them are supply links which consists of pipelines carrying water from supply sources to demand nodes, groundwater interaction links which represents the natural interaction between surface and groundwater bodies.

\subsection{Water Demand Module}

The aim of water demand module is to decrease water demands through different conservation techniques and limit the usage of water. Water demand is calculated based on off stream use, which is the water that is withdrawn and diverted from a source. The different water demand types are domestic demand, industrial demand, agricultural demand, demand for hydropower, environmental demand and demand for tourism. This paper focuses on residential domestic demand. The water demand for urban population includes demand in houses for drinking, kitchen, cooking, toilet use, and out-of house for gardening, car washing etc. Number of households and population growth rate are the two important points to be considered while calculating water demand. The most important factor for water demand estimation is permanent population

Water demand for each region is computed based on its usage type principally residential, number of occupants, usage of bore well and usage of harvested rain water. The water demand $\left(D_{b}\right)$ for building $b$ in litres per capital day (lpcd) is computed as shown in (1).

$$
D_{b}=c^{i} p^{b}
$$

Where $\mathrm{D}_{\mathrm{b}}$ is the demand for water from building $\mathrm{b}$ in lpcd; $\mathrm{p}^{\mathrm{b}}$ is the number of people expected in building $b$; $c^{i}$ is the demand coefficient per person at level i; The coefficient value is selected based on the specific kind of building and the usage of bore well and harvested rain water [1]. For example the per capita minimum water supply is $135 \mathrm{lpcd}$. The coefficient value can vary based on different geographical regions.

\subsection{Optimal Allocation}

Optimal water resource allocation means to allocate water resources scientifically and rationally in order to achieve economic, environmental and social benefits. In the year 1995 Rainer Storn and Kenneth Price proposed a new populationbased optimization technique called differential evolution algorithm to solve complex optimization problems. The complexity of genetic operation can be reduced using differential evolution algorithm by real-number encoding, continuing the use of population-based global search technique, differential-based mutation operation, keeping track the dynamics of the current search, one-to-one competition survival technique, adjusting the search strategy and achieve good global convergence and robustness without resorting to any information characteristic of the problem in question [12]. Differential Evolution uses mutation as a search mechanism and selection to direct the search toward the prospective regions in the feasible region. It is a population based search technique which utilizes NP variables as population of $\mathrm{D}$ dimensional parameter vectors for each generation. Differential evolution algorithm is used to optimize real parameter, real valued functions. The different steps in optimization algorithm are initialization, mutation, recombination and selection. The differential evolution algorithm is given as follows

Step 1: Select target vector

Step 2: Randomly select two population members

Step 3: Construct weighted difference vector

Step 4: Add a third randomly chosen vector

Step 5: Obtain trial vector using cross over with target vector Step 6: Move to next generation with smaller cost value.

In water system design evaluation of residential water demands is necessary to obtain basic information about water demands. The maximum day demand depends on water system source, treatment, and storage facilities. It is not possible to meet the maximum day demand from one source; therefore multiple sources are considered. Differential evolution algorithm is used in this paper to optimally allocate water resources.

\subsubsection{Objective Function}

The objective of optimal water allocation is to minimize the volume of regional water allocation so that the water is allocated fairly to all the users. The objective function is described using (2), subject to the following constraints.

$$
f(x)=\min \left(\sum_{k=1}^{K} \sum_{j=1}^{j(K)}\left(D_{j}^{k}-\left(\sum_{i=1}^{i(K)} x_{i j}^{k}+\sum_{m=1}^{M} x_{m j}^{k}\right)\right)\right)
$$

Let us assume that the research district has $\mathrm{k}$ sub divisions. Independent water sources for each subdivision is represented using $\mathrm{i}(\mathrm{k})$ and the water users by $\mathrm{j}(\mathrm{k})$. If $\mathrm{m}$ represent the public water sources. The decision variables $x_{i j}{ }^{k}$ and $x_{m j}{ }^{k}$ represents the volume of water allocated in sub-division $\mathrm{k}$ from independent water sources $i$ and public water sources $m$ to user $\mathrm{j}$. $\mathrm{D}_{\mathrm{j}}^{\mathrm{k}}$ represents user $\mathrm{j}$ 's water demand in sub division $\mathrm{k}$.

\subsubsection{Constraints}

The constraints associated with the amount of water supply is given in (3) and (4).

$$
\sum_{i=1}^{i(K)} x_{i j}^{k} \leq W_{i}^{k}
$$

Where $\mathrm{W}_{\mathrm{i}}^{\mathrm{k}}$ represents the upper limit of water supply in subdivision $\mathrm{k}$ from independent water source $\mathrm{i}$.

$$
\sum_{m=1}^{M} x_{m j}^{k} \leq W_{m}^{k}
$$


Where $\mathrm{W}_{\mathrm{m}}{ }^{\mathrm{k}}$ represents the upper limit of water supply in subdivision $\mathrm{k}$ from public water source $\mathrm{m}$. The constraint associated with the consumption of water is given in (5).

$$
w(c, k) \leq P_{m}^{k}
$$

Where $\mathrm{P}_{\mathrm{m}}{ }^{\mathrm{k}}$ denotes the upper limit of water consumption of public water source $\mathrm{m}$ in sub division $\mathrm{k}$. The constraints associated with the change in supply and demand of water consumption is given in (6).

$$
L_{j}^{k} \leq \sum_{i=1}^{i(K)} x_{i j}^{k}+\sum_{m=1}^{M} x_{m j}^{k} \leq H_{j}^{k}
$$

Where $L_{j}{ }^{k}$ denotes the lower limit of water-demand change of user $\mathrm{j}$ in sub division $\mathrm{k}$ and $\mathrm{H}_{\mathrm{j}}^{\mathrm{k}}$ denotes the higher limit of water-demand change of user $\mathrm{j}$ in sub division $\mathrm{k}$. Non negative constraints is given in (7) [12].

$$
x_{i j}^{k}, x_{m j}^{k} \geq 0
$$

\subsection{Water Sources and Water Availability}

The water needed to supply to an urban population is taken from a water source. The most common sources of water include reservoirs, lakes, rivers and groundwater. In this study the different water sources available to the domestic users of Bengaluru are from the Cauvery reservoir, bore wells and usage of harvested rain water. Water availability (GEMI, 2012) is defined as the hydrologic capacity of a water source like municipal water, groundwater and surface water body to sustain additional water demands after considering other current water uses and water conditions. The availability of water changes from year to year, or even between months. Water supply is based on the availability of water and the capacity of the facility used to withdraw water from the water source.

\section{CONCLUSION}

Evolutionary algorithms are suitable for optimization techniques based on natural evolution. Therefore in this paper for water optimization differential evolution algorithm is used. Water use efficiency is the criteria used for framing the allocation policy. The objective is to encourage domestic users to meet their increasing demand by recycling and reusing or depend on other sources of water. The model is intended to serve as a diagnosis tool to facilitate discussions and cooperation among different domestic water users and upstream/downstream stakeholders to make informed decisions for sustainable and equitable water resources development. Traditional urban centralized architecture was based on supply and disposal but here the focus is on hybrid systems where municipal supply is amplified with building level water provisioning via, usage of bore wells and rainwater harvesting. Adjustments to design criteria are incorporated to reflect actual conditions. The model incorporates variations in demand that can occur due to the changes in water use patterns. Industrial, commercial, or other nonresidential water demands can also be incorporated into the future allocation model. Sewage water treatment, water demand variations based on seasons, availability and water loss due to leakage and pump failure are considered in the future models. This model is also extended to be used as an illustrative analysis of allocation of water for water data in different cities.

\section{REFERENCES}

[1] Afreen Siddiqi, Olivier L. de Weck, 2013, Quantifying End-Use Energy Intensity of the Urban Water Cycle, Journal Of Infrastructure Systems $\odot$ Asce.

[2] J. J. YOU, Z. GAN, H. GAN and L. JIA, 2011, Development and application of water allocation model based on ET-control, Risk in Water Resources Management ,Proceedings of Symposium H03 held during IUGG2011 in Melbourne, Australia.

[3] Priya Singh, Pankaj Deep Kaur, 2017, Review on Data Mining Techniques for Prediction of Water Quality, International Journal of Advanced Research in Computer Science.

[4] Mahmood A. Khan,Md Zahidul Islam, Mohsin Hafeez, 2012, Evaluating the Performance of Several Data Mining Methods for Predicting Irrigation Water Requirement, Proceedings of the Tenth Australasian Data Mining Conference, Sydney, Australia.

[5] Patel S.S, Ramachandran P, 2017, Sustain. Water Resour. Manag.

[6] Stephen X. Zhang and Vladan Babovic, 2012, A real options approach to the design and architecture of water supply systems using innovative water technologies under uncertainty, Journal of hydro informatics.

[7] M. S. Babel, A. Das Gupta and D. K. Nayak, 2005, A Model For Optimal Allocation Of Water To Competing Demands, Water Resources Management.

[8] Ahmad I, Zhang F, Liu J, Anjum M N, Zaman M, Tayyab M, et al, 2018, A linear bi-level multi-objective program for optimal allocation of water resources, https://doi.org/10.1371/journal.pone.0192294

[9] Ling H, Gui F, 2009, Applying genetic algorithm in optimal allocation of water resources based on water rights, Control and Decision Conference, IEEE.

[10] Roozbahani R, Schreider S, Abbasi B, 2013, Multiobjective decision making for basin water allocation, Modelling and Simulation Society of Australia and New Zealand.

[11] https://www.researchgate.net/publication/265117611_kar nataka_state_water_sector_reform_current_status_emerg ing_issues_and_needed_strategies. http://www.bwssb.org/sites/default/files/bwssb-newtariff.pdf.

[12] K. P. Feng and J. C. Tian, 2013, Water Resources Optimal Allocation Based on Multi-Objective Differential Evolution Algorithm, Applied Mechanics and Materials, Vols. 278-280. 